\title{
CUANTIFICACIÓN DE AGUA Y CARBONO EN PLANTACIONES DE TECA (Tectona grandis, L.F.) EN BAHIA DE BANDERAS, NAYARIT, MÉXICO.
}

\author{
Agustin Gallegos R. ${ }^{1}$, Agustina Garcia $\mathrm{O}^{2}$., Bernardino Parada $\mathrm{S}^{3}$., \\ Juan de Dios Benavides ${ }^{4}$, Efrén Hernández A. ${ }^{5}$ y José R. Gómez Aguilar ${ }^{6}$
}

\section{RESUMEN}

Los servicios ambientales son aquellos procesos y funciones de los ecosistemas que, además de influir directamente en el mantenimiento de la vida, generan beneficios y bienestar para las personas y las comunidades, pero es necesario evaluarlos para conocer el efecto real que tienen los bosques y plantaciones en su preservación. En este trabajo se presentan los resultados de la cuantificación del agua y carbono en una plantación de teca.

En el año 2005 se estableció un experimento en tres plantaciones comerciales de teca (Tectona grandis L. F.) de 8, 9 y 10 años de edad en el municipio de Bahia de Banderas, Nayarit, con el propósito de cuantificar la distribución del agua de lluvia. En la plantación de 8 años se determino la producción de biomasa y captura de carbono. Para conocer la distribución de la lluvia relacionada con la plantación se instalaron pluviómetros en áreas fuera de la plantación y dentro de la plantación con la finalidad de conocer la precipitación total, precipitación interfoliar y el escurrimiento fustal. El periodo de medición en el año 2005 fue del 10 de julio al 15 de octubre, la toma de datos fue semanal, haciendo un total de 14 periodos de observación. Los resultados muestran que durante el periodo la precipitación total fue de $1.077 \mathrm{~mm}$, la precipitación interfoliar total fue de $815 \mathrm{~mm}$ y el flujo fustal fue de $103 \mathrm{~mm}$, correspondiendo un $75 \%$ y un $10 \%$ a la precipitación interfoliar y flujo fustal, respectivamente. La intercepción obtenida de los datos anteriores representa $159 \mathrm{~mm}$ y corresponde a un porcentaje del $15 \%$ con respecto a la lluvia total, valor que se encuentra dentro del limite inferior de árboles de hoja ancha. Estos valores indican que las perdidas por intercepción no son altas y se esperaria que una gran cantidad de agua producto del flujo interfoliar y del tallo se ingresaria al suelo a través de la infiltración, propiciando recarga de mantos freáticos.

El potencial de captura de carbono se obtuvo en la plantación de 8 años de edad, en la cual se realizaron derribos de árboles para conocer el peso seco de la biomasa de los árboles. Los resultados indican que la biomasa seca por árbol fue de $58,9 \mathrm{~kg}$, valor que corresponde a una captura de $\mathrm{CO}_{2}$ de $25,1 \mathrm{~kg}$. La densidad de la plantación es de 1.111 árboles/ha y la captura total de carbono actual es de $27.935 \mathrm{~kg} / \mathrm{ha}$ y una captura de carbono promedio anual

1-Profesor Investigador del centro Universitario en Ciencias Biológico Agropecuarias de la Universidad de Guadalajara, Jalisco.gra09526@cucba.udg.mx

2-Estudiante de Doctorado en Ciencias Biológico Agropecuarias, en la Universidad Autónoma de Nayarit. garciaoforestadora@hotmail.com

3-Estudiante de la Maestria tecnológica del Colegio de Postgraduados. Montecillos Mexico

4-Investigador del INIFAP. Pacifico-Centro

5-Profesor Investigador del centro Universitario en Ciencias Biológico Agropecuarias de la Universidad de Guadalajara Jalısco

6-Profesor Investigador de la Universidad Autonoma de Nayarit. roberto@nayar.uan.mx 
de $3.492 \mathrm{~kg} / \mathrm{ha}$. Valores que se consideran altos para una plantación de esas edades con otras especies.

Palabras clave: Tectona grandis, Carbono, Servicios Ambientales.

\section{CARBON AND WATER ASSESSMENT ON TECA (Tectona grandis, L.F.) PLANTED FORESTS IN BAHÍA DE BANDERAS, NAYARIT, MÉXICO.}

\section{SUMMARY}

Environmental services are those ecosystem processes and functions that besides influencing the maintenance of life, they generate comfort and goods for the people and communities. Because of the implication it is important to know the real effect of the forest and plantations to preserve these services. This work has the intention to show the results of the assessment of water and carbon in commercial plantations of teca.

During 2005 several experimental sites were established under plantations of Teca (Tectona grandis L. F.) in Bahia de Banderas, Nayarit. The age of the plantations was of 8, 9, and 10 years old and they were used for water evaluation. The biomass and carbon sequestration was only evaluated at the plantation of 8 years old. The water was measured using rain gauges in three ways, rainfall, throughfall and stemflow. The rain gauges used for rainfall were over clear stands outside the plantation and the throughfall and stemflow was directly obtained under the trees. The measured rainy season during 2005 started on July $10^{\text {th }}$ and ended on October $15^{\text {th }}$. The data was collected over weekly periods, a total of 14 periods were observed. The results show that the total rainfall was $1,077 \mathrm{~mm}$, the throughfall was $815 \mathrm{~mm}$ and stemflow 103 $\mathrm{mm}$, corresponding a $75 \%$ and $10 \%$ of throughfall and stemflow, respectively related to the total rain. The interception obtained from this data was $159 \mathrm{~mm}$ and represents a value of $15 \%$ of the total rain, value considered within the range of long leaves forest. This data show that the interception losses are not high and it is expected that most of the water from throughfall and stemflow reaches the soil and infiltrates, which after a period of time percolates reaching the aquifers.

The potential of carbon sequestration was obtained from the 8 years old plantation. The trees were cut to obtain the dry weight of the biomass. The results show that the dry biomass by tree was $58.9 \mathrm{~kg}$, transformed to $\mathrm{CO}_{2}$ sequestration the value was $25.1 \mathrm{~kg}$. The tree density was 1,111 trees/ha, according to this density the actual carbon sequestration was $27,935 \mathrm{~kg} /$ ha and an annual value by hectare of 3,492 . These values are considered high compared to other plantations with different species. The results show that water conservation and carbon sequestration can be an important role of Teca plantations and more studies need to be continued in order to confirm the first results.

Key words: Tectona grandis, Carbon, Environmental Services. 


\section{INTRODUCCIÓN}

Los servicios ambientales son el conjunto de condiciones y procesos naturales que ofrecen las áreas naturales por su simple existencia y que la sociedad puede utilizar para su beneficio. Dentro de este conglomerado de servicios se pueden señalar la biodiversidad, el mantenimiento de germoplasma con uso potencial para el beneficio humano, el mantenimiento de valores estéticos y filosóficos, la estabilidad climática, la contribución a ciclos básicos (agua, carbono, nutrientes, otros) y la conservación de suelos, entre otros. Para el caso particular de recursos forestales, la producción de tales servicios está determinada por las caracteristicas de las áreas naturales y su entorno socioeconómico (Torres y Guevara, 2002).

Dentro de los servicios ambientales que prestan las plantaciones forestales está el secuestro y almacenamiento de $\mathrm{CO}_{2}$ (el gas más abundante de los que se encuentran en la atmósfera y ocasionan el efecto invernadero.) mitigando los efectos negativos en la calidad de aire. Además de la captura de agua o desempeño hidráulico.

El potencial de infiltración de agua de un área arbolada depende de un gran número de factores tales como: la cantidad y distribución de la precipitación, el tipo de suelo, las caracteristicas del mantillo, el tipo de vegetación y geomorfologia del área, entre otros. Esto indica que la estimación de captura de agua debe realizarse para áreas especificas y con información sobre la mayor parte de estas variables (Torres y Guevara, 2002).

En el presente trabajo se pretende determinar y cuantificar de manera preliminar la captura de carbono y el agua de lluvia que ingresa a las plantaciones de Tectona grandis, a través de metodologias para cuantificar el agua de lluvia y la corta de algunos árboles para determinar el potencial de captura de carbono en el Municipio de Bahia de Banderas, Nayarit, México.

\section{OBJETIVO}

Determinar y cuantificar el ingreso de agua y la captura de carbono en plantaciones forestales de Tectona grandis de 8,9 y 10 años de edad, durante un año de observaciones.

\section{MATERIALES Y MÉTODOS}

\section{Descripción del Área de Estudio}

Este trabajo se desarrollo en el municipio de Bahia de Banderas, Estado de Nayarit, México, ubicado en la costa occidental del pais. El clima es cálido subhúmedo, Aw2(w),con régimen de lluvia de junio a diciembre y enero, con una precipitación anual que fluctúa en el rango de 1200 a $1500 \mathrm{~mm}$. La temperatura media anual va de los $22^{\circ}$ a los $24^{\circ} \mathrm{C}$, la presencia de heladas es muy poco frecuente. La dirección predominante de los vientos es de oeste a este (Figura $\mathrm{N}^{\circ} 1$ ). 
La vegetación, en general, se encuentra basada en especies de selva baja subcaducifolia, vegetación halófila, palmares y agricultura de temporal y riego, siendo las predominantes: capomo (Brosimon alicastrum), huanacaxtle (Enterolobium cyclocarpum), papelillo (Bursera arbórea),higuera (Ficus padifolia), hule (Castilla elástica), coquito de aceite (Orbignia guayacole), mata palo (F. tecolutensis), palma de llano (Sabal rosei), coyul (Gerocomia mexicana) y jarretadera (Acacia hormiguera), entre otras.

Para desarrollar este trabajo se seleccionarán 3 parcelas experimentales (plantaciones) de 1 ha, en cada una de ellas se eligen 4 subparcelas de $100 \mathrm{~m}^{2}$ en donde se establece cada uno de los experimentos que darán respuesta a las variables para determinar el agua y carbono (Figura $\mathrm{N}^{\circ}$ 2).

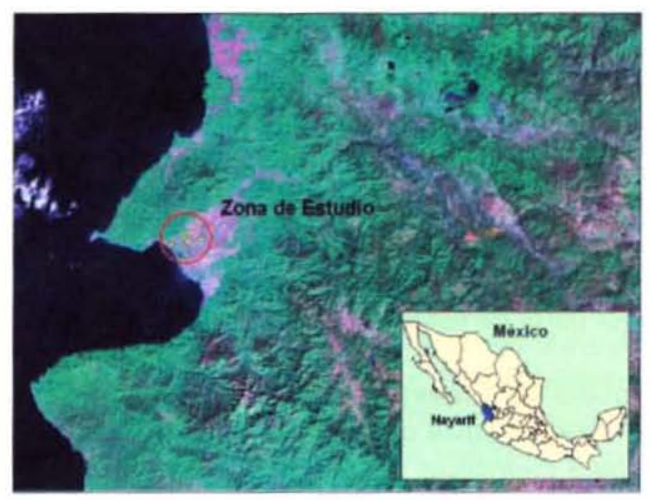

Figura $\mathrm{N}^{\circ} 1$

UBICACIÓN DEL AREA DE ESTUDIO

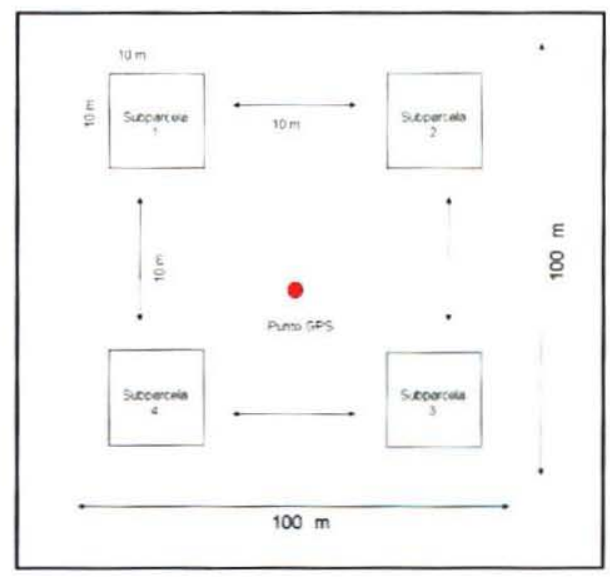

Figura $\mathrm{N}^{\circ} 2$

PARCELAS DE MUESTREO 


\section{Metodologia para Carbono}

La medición de la biomasa se efectuó de acuerdo con método propuesto por Schlegel et al. (2000). Una vez seleccionados los árboles a muestrear, se realizaron 3 tipos de mediciones, la primera con los árboles en pie, la segunda con los árboles derribados y la tercera en la parte subterránea del árbol. En el predio seleccionado se delimitó un conglomerado de 4 subparcelas de muestreo de $100 \mathrm{~m}^{2}$ cada una, (10 x $\left.10 \mathrm{~m}\right)$, a una distancia, entre punto central de cada subparcela, de $60 \mathrm{~m}$.

Todos los árboles que cayeron dentro de las subparcelas fueron medidos tomándose las siguientes variables: Diámetro a la altura del pecho (DAP), Altura a la primera rama, Altura total del árbol y Cobertura de copa (Figura $N^{\circ} 3$ ). En relación a estas variables en cada subparcela se determinó el árbol promedio, se derribó, se troceó y separaron cada uno de sus componentes (fuste, ramas y hojas) y se pesaron en fresco, esto para la parte aérea del árbol. Para la parte subterránea se extrajo la raiz, se cortó en secciones; separando raiz gruesa, medianas y chicas, se pesaron en fresco, se tomaron muestras de cada componente, se guardaron en bolsas de plástico selladas, para ser trasladadas a laboratorio, donde en una estufa se secaron a $100^{\circ}$ $\mathrm{C}$, hasta obtener su peso constante. Mediante la suma del peso fresco de cada uno de los componentes del árbol se obtuvo el peso total del árbol (PTA), y con el secado de las muestras se obtuvo, por diferencia de pesos, la cantidad de biomasa de cada árbol, determinándose en porcentaje para poder ser aplicado, porcentualmente, a todos los árboles de las subparcelas y del predio en estudio. Asimismo al peso seco de cada componente del árbol se le aplicó un indice de captura de carbono del 0.4269, propuesto por Jo y Mc Pherson (1995), ese resultado se proyectó a toda la subparcela y al predio determinándose con ello el potencial de captura de carbono atmosférico de las plantaciones forestales con la especie teca, en las condiciones del Municipio de Bahia de Banderas, México.

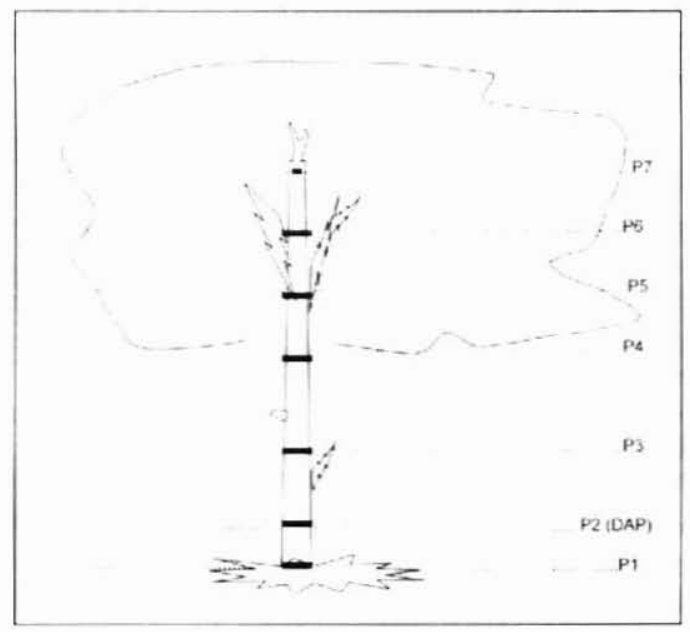

Figura $\mathrm{N}^{\circ} 3$

SECCIONES DEL ÁRBOL PARA DETERMINAR LA BIOMASA 


\section{Metodologia para Cuantificar el Ingreso de Agua}

Antes del inicio de las lluvias del verano 2005, se estableció en plantaciones forestales comerciales de teca de 8,9 y 10 años de edad un experimento para cuantificar el agua que ingresa a travès de la lluvia.

La metodologia es la descrita por Fassbender (1987), en la que se evaluan las siguientes variables: Lluvia o ingreso de agua al ecosistema, Lavaje foliar, Lavaje de tallos, Intercepción, Ingreso al suelo, Escurrimiento superficial, Evapotranspiración, Transpiración, Percolación y Egreso del suelo

Para establecer el presente experimento se seleccionaron 3 plantaciones forestales comerciales; dos de Tectona grandis (teca) y una de Cedrela Odorata (cedro). Como la superficie plantada es mayor a 1 ha, el experimento se estableció en el centro de cada plantación, a fin de evitar el efecto de orilla. El experimento se estableció de la siguiente manera:

En el centro de la parcela, se eligió una superficie de 1-00-00 ha, donde se midieron 4 subparcelas de $10 \mathrm{~m}^{2} \mathrm{c} / \mathrm{u}$, con $60 \mathrm{~m}$ de separación entre subparcela y subparcela (ver fig. 2).

En cada una de las subparcelas se estableció lo siguiente:

- Pluviómetro de material plástico bajo el dosel, prefabricado con material pvc de $2,5 \mathrm{~cm}$ de diámetro y $50 \mathrm{~cm}$ de largo, mismo que fue colocado en el interior de otro tubo pvc de $50 \mathrm{~cm}$, con diámetro de $4 \mathrm{~cm}$, que fue enterrado $20 \mathrm{~cm}$ y llenado de grava otros $20 \mathrm{~cm}$, para en los $10 \mathrm{~cm}$ restantes introducir el pluviómetro y elevarlo del suelo para evitar salpicadura de agua e introducción de particulas de suelo. Esto hace un total de 4 pluviómetros por parcela, con los que se medirá la variable de Lavaje foliar bajo el rodal (LFR). Las observaciones se realizarán en forma semanal.

- Primeramente, se midió el diámetro de todos los árboles que se encontraron en las subparcelas de $10 \mathrm{~m}^{2}$ y se les sacó una media, esta media se consideró para seleccionar 1 árbol en el centro de cada subparcela, al cual se le colocó plástico negro para acolchar desde la altura de $1 \mathrm{~m}$, sujetado con rafia. Luego se realizó una excavación con $10 \mathrm{~cm}$ de profundidad aproximadamente alrededor del árbol, para formar la canaleta con el mismo plástico con que fue cubierto el tallo: posteriormente se realizó un hoyo donde fue colocado un recipiente de $20 \mathrm{~L}$, donde se introdujo la punta de la canaleta de plástico que conducirá el agua hacia el recipiente donde se medirá la variable Lavaje de Tallos (LT) y las observaciones serán semanales.

- Se coloco 1 canaleta de $1 \mathrm{~m}^{2}$ para delimitar el área donde se medirá el escurrimiento superficial, la canaleta fue construida con 4 tablas de madera de $1 \mathrm{~m}$ de largo y $20 \mathrm{~cm}$ de ancho, en un extremo (del lado de la pendiente de cada terreno) se realizó un orificio de $2 "$, donde se colocó un tubo de PVC de $15 \mathrm{~cm}$ de largo que conducirà el agua escurrida hacia un colector de plástico de $20 \mathrm{~L}$, que previamente fue enterrado. En este colector se medirá la variable Escurrimiento superficial del suelo (ESS) mediante observaciones 
semanales.

- En cada subparcela, se realizará un perfil de humedad, muestreando a 20,40 y 60 $\mathrm{cm}$ de profundidad, utilizando el método gravimétrico. Posteriormente se realizará la transformación a láminas de agua, para conocer el \% obtenido. Estos muestreos se realizarán en forma mensual, con lo que se determinará la variable Percolación (PER).

- Finalmente, en la periferia de la plantación (fuera del rodal), se colocaron 4 pluviómetros similares a los antes descritos para medir el agua de lluvia o el Ingreso de agua al ecosistema (IAE) mediante observaciones semanales.

Variables estimadas:

Intercepción; equiparable a la evaporación desde el rodal:

Se calculará como la diferencia entre la lluvia y la suma del lavaje foliar y de los tallos, la fórmula es la siguiente:

$$
(E V R)(I)=\sum I A E-\left(\sum L F+\sum L T\right)
$$

Donde:

$$
\begin{aligned}
& \text { EVR = Evaporación } \\
& I=\text { Intercepción } \\
& \sum I A E=\text { Suma de ingreso de agua al ecosistema } \\
& \sum L F=\text { suma de lavaje foliar } \\
& \sum L T=\text { suma de lavaje de tallos }
\end{aligned}
$$

Ingreso al suelo:

Se calcula como la suma del lavaje foliar y el lavaje de tallos, mediante la ecuación siguiente:

$$
\mathbf{I A S}=\sum \mathbf{L F}+\sum \mathbf{L T}
$$

Donde:

$$
\begin{aligned}
& \text { IAS }=\text { Ingreso de agua al suelo } \\
& \sum \mathrm{LF}=\text { suma de lavaje foliar } \\
& \sum \mathrm{LT}=\text { suma de lavaje de tallos }
\end{aligned}
$$

\section{Evapotranspiración:}

Se determinará con los datos climáticos que por evaporación se reporten en la estación meteorológica de San José del Valle Nayarit., utilizando la fórmula de Blaney y Cridley.

Transpiración del rodal, equiparable a la absorción de agua a partir del suelo:

Se calcula como la diferencia entre la evapotranspiración y la evaporación (intercepción) del rodai. 


\section{RESULTADOS Y DISCUSIÓN}

Los resultados son obtenidos de 4 subparcelas con 34, 27, 24 y 27 árboles, haciendo un total de 112, de los cuales sólo se derribaron 4 para el cálculo de la biomasa. En el Cuadro $\mathrm{N}^{\circ}$ 1 se presenta la relación de Peso Seco / Peso fresco por cada árbol muestra.

\section{Cuadro $N^{\circ} 1$}

POTENCIAL DE CAPTURA DE CARBONO POR ÁRBOL MUESTRA

\begin{tabular}{|l|c|c|c|c|}
\hline & ARBOL 13 & ARBOL 27 & ARBOL 13 & ARBOL 21 \\
\hline PFTA (kg) & 80.415 & 58 & 178.28 & 168.145 \\
\hline PS $\mathbf{( k g )}$ & 33.0808 & 31.001 & 85.289152 & 87.4354 \\
\hline$\%$ & 41.13 & 53.45 & 47.84 & 52.00 \\
\hline Carbono capturado (kg) & 14.122 & 13.234 & 36.409 & 37.326 \\
\hline$\%$ & 17.56 & 22.81 & 20.42 & 22.19 \\
\hline
\end{tabular}

PFTA $=$ peso fresco total árbol

$\mathrm{PS}=$ peso seco

Estableciendo una proyección, sumando las proporciones de peso seco (PS) por cada árbol muestreado, arroja un indicador que puede ser aplicado a las subparcelas de muestreo y al predio en general, con ello se determina su potencial para el Peso Seco total y aplicando el indice de Jo y Mc Pherson se obtiene el potencial del secuestro de carbono (SC). (Cuadro $\mathrm{N}^{\circ}$ 2).

Indicador proyectado de peso seco

$$
\mathrm{PS}=(41.13+53.45+47.84+52) / 4=48,6 \%
$$

\section{Cuadro $\mathrm{N}^{\circ} 2$}

PROYECCIÓN DE PESO SECO Y CARBONO SECUESTRADO POR CADA SUBPARCELA

\begin{tabular}{|c|c|c|c|c|c|}
\hline Subparcela & $\begin{array}{c}\mathbf{N}^{0} \text { de } \\
\text { árboles }\end{array}$ & $\begin{array}{c}\text { Peso del árbol } \\
\text { promedio }\end{array}$ & $\begin{array}{c}\text { PF total de la } \\
\text { subparcela }\end{array}$ & $\begin{array}{c}\text { PS total de la } \\
\text { subparcela }\end{array}$ & $\begin{array}{c}\text { SC total de la } \\
\text { subparcela }\end{array}$ \\
\hline 1 & 34 & 80.415 & 2412.45 & 1172.4507 & 500.52 \\
\hline 2 & 27 & 58.00 & 1566 & 761.076 & 324.90 \\
\hline 3 & 24 & 178.28 & 4278.72 & 2079.4592 & 887.72 \\
\hline 4 & 27 & 168.145 & 4539.915 & 2206.3986 & 941.91 \\
\hline TOTAL & 112 & 484.84 & 12797.085 & 6219.3845 & 2655.05 \\
\hline
\end{tabular}

Donde: $\mathrm{PF}=$ peso fresco, $\mathrm{PS}=$ peso $\mathrm{seco}, \mathrm{SC}=$ secuestro de carbono

Como las parcelas fueron seleccionadas de manera aleatoria, siguiendo la misma metodologia, se puede determinar un promedio de peso fresco total para todos los árboles, que en este caso seria de 121,21 kg y aplicando el Indicador Proyectado de Peso Seco de 48,6, daria un peso seco de $58,9 \mathrm{~kg}$ y multiplicándolo por el indice de Jo y Mc Pherson arrojaria que 
la captura de carbono por ese árbol promedio sería de 25,1478 kg de carbono secuestrado y multiplicándolo por el total de árboles del predio (1.111 árboles/ha) arroja la cantidad de 27.939 $\mathrm{kg} / \mathrm{ha}$ a los 8 años de edad. Cantidad que según lo reportado por otros autores es buena.

Se observó que el diámetro de las raices, en promedio, fue de 2,5 m, dato a considerar para próximas extracciones. El sistema radicular de teca es extendido y una raiz de las principales se anclaba con mayor profundidad y siempre en dirección al oriente, la dirección en que corren los vientos venidos de la bahia.

Los resultados de la cuantificación del ingreso de agua de las plantaciones de teca arrojaron los siguientes:

- $\quad$ El periodo de medición en el año 2005 fue del 10 de julio al 15 de octubre, la toma de datos fue semanal, haciendo un total de 14 periodos de observación (Cuadro $\mathrm{N}^{\circ}$ 3). Los resultados muestran que durante el periodo la precipitación total fue de $1.077 \mathrm{~mm}$, la precipitación interfoliar total fue de $815 \mathrm{~mm}$ y el flujo fustal fue de 103 $\mathrm{mm}$, correspondiendo a un $75 \%$ y un $10 \%$ a la precipitación interfoliar y flujo fustal, respectivamente. La intercepción obtenida de los datos anteriores representa 159 $\mathrm{mm}$ y corresponde a un porcentaje del $15 \%$ con respecto a la lluvia total, valor que se encuentra dentro del limite inferior de árboles de hoja ancha. Estos valores indican que las perdidas por intercepción no son altas y se esperaria que una gran cantidad de agua producto del flujo interfoliar y del tallo se ingresaria al suelo a través de la infiltración, propiciando recarga de mantos freáticos.

\section{Cuadro $\mathrm{N}^{\circ} 3$}

PROMEDIOS MENSUALES (mm) DURANTE EL PERIODO DE LLUVIA 2005

\begin{tabular}{|l|c|c|c|c|}
\hline \multirow{2}{*}{\multicolumn{1}{|c|}{ VARIABLE }} & \multicolumn{3}{c|}{ PROMEDIOS MENSUALES } \\
\cline { 2 - 5 } & JUL & AGO & SEP & OCT \\
\hline IAE (Ingreso de agua al ecosistema) & $\mathbf{2 7 6 . 7 5}$ & $\mathbf{3 8 7 . 0 3}$ & $\mathbf{2 8 6 . 4 0}$ & $\mathbf{1 2 6 . 4}$ \\
\hline $\mathrm{LF}$ (Lavaje foliar) & 192.12 & 293.54 & 228.96 & 100.8 \\
\hline $\mathrm{LT}$ (Lavaje de tallos) & 24.82 & 36.48 & 28.67 & 12.6 \\
\hline ESS (Escurrimiento superficial del suelo) & 83.60 & 116.11 & 86.42 & 37.82 \\
\hline I (Intercepción) & 59.81 & 57.01 & 28.77 & 12.96 \\
\hline Is (Ingreso al suelo) & 216.94 & 330.02 & 257.63 & 113.41 \\
\hline Et (Evapotranspiración) & 122.4 & 128.7 & 103.5 & 104.7 \\
\hline $\mathrm{Tr}$ (Transpiración) & 30.9 & 32.2 & 26.1 & 26.2 \\
\hline $\mathrm{P} \quad$ (Percolación) & 7.7 & 19.0 & 27.1 & 31.0 \\
\hline
\end{tabular}

\section{CONCLUSIONES}

Los resultados preeliminares obtenidos de captura de carbono de las plantaciones de teca de 8 años, comparados con reportes de otros autores, son buenos. Con respecto a los datos del balance hídrico, la intercepción reporta un valor que se encuentra dentro del limite 
inferior de árboles de hoja ancha. Estos valores indican que las perdidas por intercepción no son altas y se esperaria que una gran cantidad de agua producto del flujo interfoliar y del tallo se ingresaria al suelo a través de la infiltración, propiciando recarga de mantos freáticos

Es importante mencionar que dentro de este estudio, se analizará las ventajas y desventajas que tienen los servicios de agua y carbono en plantaciones comerciales en comparación con los bosques nativos, para tratar de evaluar si las plantaciones son una opción en términos de los servicios ambientales.

\section{RECONOCIMIENTOS}

Agradecimiento al Proyecto CONACYT-CONAFOR 2004-CO4-57

\section{REFERENCIAS}

Comisión Nacional del Agua, 2005. Registro de Información Climática de la Estación San José del Valle, Nayarit a partir de 1979.

Escobedo R. J. S., 1983. Calibración de un pluviómetro de poliducto, con otros de tipo comercial. Ponencia presentada en el XVI Congreso Nacional de la S.M.C.S. Oaxaca, Oax. Terra. Año 2, No. 1. 1984. Pág. 80-84.

Garcia P, J. D., et al., 1991. Calibración de dos tubos PVC para su uso como pluviómetros. V. Congreso Nacional de Meteorologia. Cd. Juárez, Chihuahua. Memoria. Pág. 127.

Fassbender H. W., 1987. Modelos edafológicos de sistemas agroforestales. Centro Agronómico Tropical de Investigación y Enseñanza, CATIE. Turrialba, Costa Rica. 475 p. (Serie de materiales de enseñanza No. 29).

INEGI, 2000. Síntesis de Información Geográfica del Estado de Nayarit,

Jo H. E. y E. G. Mc Pherson, 1995. Carbon storage and flux in urban residencial greenpace. J. Env. Mgmt. 45:109-103

Parada, Bernardino, 2000. Las plantaciones forestales comerciales de Teca (Tectona grandis L.f) una alternativa de desarrollo para la región costera en el estado de Nayarit, Tesis de Maestria ,Chapingo, Mexico.

Roberto Sanquetta, Farinha Watzlawic, L. y Eduardo Arce J., s/f. Ecuaciones de biomasa aérea y subterránea en plantaciones de Pinus taeda en el sur del Estado de Paraná, Brasil", Universidad Federal de Paraná, Departamento de Ciencias Forestales.

Schlegel, B., Gayoso J. y Guerra J., 2000. Manual de Procedimientos, muestreo de biomasa forestal. Universidad Austral de Chile, Valdivia.

Torres Rojo Juan Manuel y Guevara Sanginés Alejandro, 2002. El potencial de México para la producción de servicios ambientales: captura de carbono y desempeño hidráulico.Gaceta Ecologica, Instituto nacional de ecologia. En: http://redalyc.uaemex.mx/redalyc/ pdf/539/53906303.pdf 